\title{
Globale Stability in a Viral Infection Model with Beddington-DeAngelis Functional Response
}

\author{
Wang Zhanwei* and He Xia
}

Department of Mathematics and Physics, Zhengzhou Institute of Aeronautical Industry Management, Henan, Zhengzhou, 450015, P.R. China

\begin{abstract}
The stability of a mathematical model for viral infection with Beddington-DeAngelis functional response is considered in this paper. If the basic reproduction number $R_{0} \leq 1$, by the Routh-Hurwitz criterion and Lyapunov function, the uninfected equilibrium $E_{0}$ is globally asymptotically stable. Then, the global stability of the infected equilibrium $E_{1}$ is obtained by the method of Lyapunov function.
\end{abstract}

Keywords: Beddington-DeAngelis, equilibrium, Global dynamics, HIV model.

\section{INTRODUCTION}

Human Immunodeficiency Virus and Acquired Immune Deficiency Syndrome (AIDS) have received much attention from the first case of AIDS was diagnosed on December 1st in 1981. It is proven to be valuable in understanding the population dynamics of viral load in vivo with mathematical models. In the last decade, many mathematical models have been developed to describe the infection with Human Immunodeficiency virus (HIV) (see[1-9]). Nowak et al. [1, 3] proposed the following model:

$$
\left\{\begin{array}{l}
\frac{d x}{d t}=\lambda-d x-\beta x v, \\
\frac{d y}{d t}=\beta x v-a y, \\
\frac{d v}{d t}=k y-r v .
\end{array}\right.
$$

where $x(t), y(t)$ and $v(t)$ represent the numbers(densities) of healthy $C D 4^{+} \mathrm{T}$-cells, infected $C D 4^{+} \mathrm{T}$-cells and viral particles at time $t$ respectively. The model assumed that healthy $C D 4^{+}$T-cells are infected at a rate $\beta x v$, infected $C D 4^{+} \mathrm{T}$-cells are lost at a rate $a y$, and virus are produced by infected $C D 4^{+} \mathrm{T}$-cells at a rate $k y$ and removed at a rate $r v$. In model (1.1), it is also assumed that healthy $C D 4^{+} \mathrm{T}$-cells are input at a constant rate $\lambda$, and die at a rate $d x$.

Although the infection rate is bilinear in most HIV-I models with the virus $v$ and healthy $C D 4^{+} \mathrm{T}$ - cells $x$, actual incidence rates are probably not linear over the entire range of $v$ and $x$. Thus, it is reasonable for our paper to

*Address correspondence to this author at the Department of Mathematics and Physics, Zhengzhou Institute of Aeronautical Industry Management, Henan, 450015, P.R. China; Tel: 13838523683;

E-mail: zziawangzhanwei@163.com assume that the infection rate of the form $\frac{\beta x v}{1+m x+n v}$, where $m, n \geq 0$ are constants. The function response $\frac{\beta x v}{1+m x+n v}$ was introduced by Beddington [10] and DeAngelis et al. [11].

In this paper, we consider a HIV-I model with Beddington-DeAngelis function response as follows:

$$
\left\{\begin{array}{l}
x^{\prime}=\lambda-d x-\frac{\beta x z}{1+m x+n z}, \\
y^{\prime}=\frac{\beta x z}{1+m x+n z}-a y, \\
z^{\prime}=k y-b z .
\end{array}\right.
$$

The biological meanings of these parameters are the similar to those appearing parameters in model (1.1).

\section{EQUILIBRIA AND GLOBAL STABILITY ANALYSIS}

Let

$R_{0}=\frac{\beta \lambda k}{(d+m \lambda) b a}$

Then $R_{0}$ is the basic reproductive number of model (1.2), which describes the average number of newly infected $\mathrm{T}$-cells generated from one infected T-cells. We can obtain that $E_{0}=\left(x_{0}, 0,0\right)$ is an uninfected equilibrium where $x_{0}=\lambda / d$, and $E_{1}=\left(x_{1}, y_{1}, z_{1}\right)$ is a infected equilibrium of model (1.2) if and only if $R_{0}>1$ where 


$$
\begin{aligned}
& x_{1}=\frac{b a+n k \lambda}{\beta k+n k d-b a m}, \quad y_{1}=\frac{\lambda \beta k-\lambda b a m-d b a}{a(\beta k+n k d-b a m)}, \\
& z_{1}=\frac{k(\lambda \beta k-\lambda b a m-d b a)}{b a(\beta k+n k d-b a m)}
\end{aligned},
$$

Now, we begin to study the stabilities of these two equilibria.

Firstly, we begin to study the stability of the uninfected equilibrium $E_{0}=\left(x_{0}, 0,0\right)$. Evaluating the Jacobian matrix of model (1.2) at $E_{0}$ gives

$$
J\left(E_{0}\right)=\left[\begin{array}{ccc}
-d & 0 & -\frac{\beta \lambda}{d+m \lambda} \\
0 & -a & \frac{\beta \lambda}{d+m \lambda} \\
0 & k & -b
\end{array}\right] .
$$

By simple computations, the characteristic equation is

$$
\begin{aligned}
\Delta(U) & =U^{3}+(a+d+b) U^{2}+\left(a d+b d+a b-\frac{k \beta \lambda}{d+m \lambda}\right) U+a d b-\frac{k \beta \lambda}{d+m \lambda} d \\
& =U^{3}+A_{1} U^{2}+A_{2} U+A_{3}
\end{aligned}
$$

Where $A_{1}=a+d+b>0, A_{2}=a d+b d+a b-\frac{k \beta \lambda}{d+m \lambda}>0$,

$$
\begin{aligned}
A_{3}=a d b- & \frac{k \beta \lambda}{d+m \lambda} d>0, \text { and } \\
A_{1} A_{2}-A_{3}= & a^{2}(b+d)+b^{2}(a+d)+d^{2}(a+b)+2 a b d \\
& -\frac{k \beta \lambda}{d+m \lambda}(a+b)>0
\end{aligned}
$$

if $R_{0}<1$. According to the Routh-Hurwitz criterion, it is obtained that uninfected equilibrium $E_{0}$ is locally asymptotically stable when $R_{0}<1$.

When $R_{0}>1$ it is easy to obtain that $A_{3}<0$, and $\Delta(0)=A_{3}<0, \Delta(+\infty)=+\infty$. That is to say the characterristic equation has positive solution. So $E_{0}$ is unstable when $R_{0}>1$.

Moreover, we construct a Lyapunov function for studying the global stability. Let

$L_{0}(x, y, z)=\frac{1}{1+m x_{0}}\left(x-x_{0}-x_{0} \ln \frac{x}{x_{0}}\right)+y+\frac{a}{k} z$

Along the trajectories of model (1.2),

Because $2-x_{0} / x-x / x_{0} \leq 0$, if $R_{0} \leq 1$ it can be obtained $\frac{d L_{0}(x, y, z)}{d t} \leq 0$ for all $x>0, y>0, z>0$. Furthermore, when and only when $x=x_{0}, y=0, z=0$, we have $\left.\frac{d L_{0}}{d t}\right|_{\left(x_{0}, 0,0\right)}=0$. By the LaSalle invariance principle, when $R_{0} \leq 1$ the uninfected equilibrium $E_{0}$ is globally asymptotically stable.
Summarizing the discussion above, we obtained the following conclusion.

Theorem 2.1 The uninfected equilibrium $E_{0}$ is globally asymptotically stable when $R_{0} \leq 1$ and is unstable when $R_{0}>1$.

Then we begin to analysis the stability of infected equilibrium $E_{1}$. Let

$L_{1}(x, y, z)=x-x_{1}-\int_{x_{1}}^{x} \frac{a y_{1}}{\beta z_{1} t / 1+m t+n z_{1}} d t+y-y_{1}-y_{1} \ln \frac{y}{y_{1}}+\frac{a}{k}\left(z-z_{1}-z_{1} \ln \frac{z}{z_{1}}\right)$

Along the trajectories of model (1.2), we obtained

$$
\begin{aligned}
\left.L_{1}\right|_{(1.2)}= & x-x_{1}-\int_{x_{1}}^{x} \frac{a y_{1}}{\beta z_{1} t / 1+m t+n z_{1}} d t+y-y_{1}-y_{1} \ln \frac{y}{y_{1}}+\frac{a}{k}(z \\
& \left.-z_{1}-z_{1} \ln \frac{z}{z_{1}}\right) \\
= & x^{\prime}-\frac{a y_{1}\left(1+m x+n z_{1}\right)}{\beta z_{1} x} x^{\prime}+y^{\prime}-\frac{y_{1}}{y} y^{\prime}+\frac{a}{k}\left(z^{\prime}-\frac{z_{1}}{z} z^{\prime}\right) \\
= & \lambda-d x-\frac{a y_{1}\left(1+m x+n z_{1}\right)}{\beta z_{1} x}\left(\lambda-d x-\frac{\beta z x}{1+m x+n z}\right) \\
& -\frac{y_{1}}{y} \frac{\beta z x}{1+m x+n z}+a y_{1}-\frac{a b z}{k}-\frac{z_{1} a y}{z}+\frac{a b z_{1}}{k}
\end{aligned}
$$

Since $\left(x_{1}, y_{1}, z_{1}\right)$ is the equilibrium point of (1.2), we have

$$
\lambda=d x_{1}+a y_{1}, \frac{y_{1}}{z_{1}}=\frac{b}{k}, a y_{1}=\frac{\beta x_{1} z_{1}}{1+m x_{1}+n z_{1}}
$$

So

$$
\begin{aligned}
& \left.L_{0}{ }^{\prime}\right|_{(1.2)}=\frac{1}{1+m x_{0}}\left(x^{\prime}-x_{0} \frac{1}{x} x^{\prime}\right)+y^{\prime}+\frac{a}{k} z^{\prime} \\
& =\frac{1}{1+m x_{0}}\left(1-x_{0} \frac{1}{x}\right)\left(\lambda-d x-\frac{\beta x z}{1+m x+n z}\right)+\frac{\beta x z}{1+m x+n z} \\
& -a y+\frac{a}{k}(k y-b z) \\
& =\frac{1}{1+m x_{0}}\left(\lambda-d x-\frac{x_{0}}{x} \lambda+x_{0} d\right)+\frac{1}{1+m x+n z}\left(\frac{\beta x_{0} z}{1+m x_{0}}\right. \\
& \left.-\frac{\beta x z}{1+m x_{0}}\right)-\frac{a b}{k} z+\frac{\beta x z}{1+m x+n z} \\
& =\frac{x_{0} d}{1+m x_{0}}\left(2-\frac{x_{0}}{x}-\frac{x}{x_{0}}\right)+\frac{1}{1+m x+n z}\left(\frac{\beta x_{0} z}{1+m x_{0}}-\frac{\beta x z}{1+m x_{0}}\right. \\
& \left.-\frac{a b z(1+m x+n z)}{k}+\beta x z\right) \\
& =\frac{x_{0} d}{1+m x_{0}}\left(2-\frac{x_{0}}{x}-\frac{x}{x_{0}}\right)+\frac{1}{1+m x+n z}\left(\frac{m x \beta x_{0} z+\beta x_{0} z}{1+m x_{0}}\right. \\
& \left.-\frac{a b z(1+m x+n z)}{k}\right) \\
& =\frac{x_{0} d}{1+m x_{0}}\left(2-\frac{x_{0}}{x}-\frac{x}{x_{0}}\right)+\frac{1}{1+m x+n z}\left(\frac{\beta \lambda z(m x+1)}{d+m \lambda}\right. \\
& \left.-\frac{a b z(1+m x)}{k}-\frac{a b n z^{2}}{k}\right) \\
& =\frac{x_{0} d}{1+m x_{0}}\left(2-\frac{x_{0}}{x}-\frac{x}{x_{0}}\right)+\frac{a b z(1+m x)}{(1+m x+n z) k}\left(R_{0}-1\right)- \\
& \frac{a b n z^{2}}{k(1+m x+n z)}
\end{aligned}
$$


Moreover

$$
\begin{aligned}
& a y_{1}\left(1+\frac{z}{z_{1}}-\frac{z}{z_{1}} \frac{1+m x+n z_{1}}{1+m x+n z}-\frac{1+m x+n z}{1+m x+n z_{1}}\right) \\
= & a y_{1}\left(\frac{n z_{1}-n z}{1+m x+n z_{1}}+\frac{z}{z_{1}} \frac{n z-n z_{1}}{1+m x+n z}\right) \\
= & a y_{1} n\left(z_{1}-z\right)\left(\frac{1}{1+m x+n z_{1}}-\frac{z}{z_{1}} \frac{1}{1+m x+n z}\right) \\
= & a y_{1} n\left(z_{1}-z\right) \frac{z_{1}-z+m x\left(z_{1}-z\right)}{z_{1}(1+m x+n z)\left(1+m x+n z_{1}\right)} \\
= & \frac{a y_{1} n\left(z_{1}-z\right)^{2}(1+m x)}{z_{1}(1+m x+n z)\left(1+m x+n z_{1}\right)} \geq 0
\end{aligned}
$$

and

$$
\begin{aligned}
& 4-\frac{x_{1}}{x} \frac{1+m x+n z_{1}}{1+m x_{1}+n z_{1}}-\frac{x z y_{1}}{x_{1} z_{1} y_{1}} \frac{1+m x_{1}+n z_{1}}{1+m x+n z}-\frac{z_{1} y}{z y_{1}} \\
& -\frac{1+m x+n z}{1+m x+n z_{1}} \leq 0
\end{aligned}
$$

We got for all $x>0, y>0, z>0, \frac{d L_{1}(x, y, z)}{d t} \leq 0$ holds. Moreover, $\frac{d L_{1}}{d t}=0$ when and only when $x=x_{1}$, $y=y_{1}, z=z_{1}$. According to LaSalle invariance principle, the infected equilibrium $E_{1}$ is globally asymptotically stable.

Theorem 2.2 The infected equilibrium $E_{1}$ exists and is globally asymptotically stable when $R_{0}>1$.

\section{CONCLUSION}

In this paper, we have investigated a HIV-I mathematical model with Beddington-DeAngelis function response. According to the Routh-Hurwitz criterion and LaSalle invariance principle, we obtained the following conclusion: 1) when $R_{0} \leq 1$ the uninfected equilibrium $E_{0}$ is globally asymptotically stable; 2) when $R_{0}>1$ the infected equilibrium $E_{1}$ is globally asymptotically stable.

\section{CONFLICT OF INTEREST}

The authors confirm that this article content has no conflict of interest.

\section{ACKNOWLEDGEMENTS}

This work was financially supported by the Natural Science Foundation of the Education Department of Henan Province (NO14B110007)

\section{REFERENCES}

[1] Nowak MA, Bangham CRM. Population dynamics of immune responses to persistent virus. Science 1996; 272(5258): 74-9.

[2] Perelson A, Nelson P. Mathematical models of HIV dynamics in vivo. SIAM Rev 1999; 41: 3-44.

[3] Nowak MA, May RM. Virus Dynamics. New York: Oxford University press, 2000: 15-80.

[4] Yang Y, Xiao Y. Threshold dynamics for an HIV model in periodic environments. J Math Anal Appl 2010; 361(1): 59-68.

[5] Wang X, Wang W. An HIV infection model based on a vectored immunoprophylaxis experiment. J Theoret Biol 2012; 313: 127-35.

[6] $\mathrm{Xu}$ R. Global stability of a delayed epidemic model with latent period and vaccination strategy. Appl Mathemat Mode 2012; 36(11): 5293-300.

[7] Wang X, Elaiw A, Song X. Global properties of a delayed HIV infection model with CTL immune response. Appl Mathemat Comput 2012; 218(18): 9405-14.

[8] Wang X, Tao Y. Lyapunov function and global properties of virus dynamics with CTL immune response. Int J Biomathemat 2008; 1(4): 443-8.

[9] Wang X,Tao Y, Song X. A delayed HIV-I infection model with Bedington-DeAngelis functional response. Nonlinear Dyn 2010; 62(1-2): 67-72.

[10] Beddington JR. Mutual interference between parasites or predators and its effect on searching efficiency. J Animal Ecol 1975; 44(1): 331-40.

[11] DeAngelis DL, Goldstein RA, O’Neill RV. A model for trophic interaction. Ecology 1975; 56(4): 881-92.

(C) Zhanwei and Xia; Licensee Bentham Open.

This is an open access article licensed under the terms of the Creative Commons Attribution Non-Commercial License (http://creativecommons.org/licenses/by-nc/3.0/) which permits unrestricted, non-commercial use, distribution and reproduction in any medium, provided the work is properly cited. 